\title{
MULTI-SCALE CARDIOVASCULAR FLOW ANALYSIS BY AN INTEGRATED MESHLESS-LUMPED PARAMETER MODEL
}

\author{
LEONARDO A. BUENO ${ }^{1}$, EDUARDO A. DIVO ${ }^{1}$ \& ALAIN J. KASSAB ${ }^{2}$ \\ ${ }^{1}$ Department of Mechanical Engineering, Embry-Riddle Aeronautical University, Daytona Beach, FL, USA. \\ ${ }^{2}$ Department of Mechanical and Aerospace Engineering, University of Central Florida, Orlando, FL, USA.
}

\begin{abstract}
A computational tool that integrates a Radial basis function (RBF)-based Meshless solver with a Lumped Parameter model (LPM) is developed to analyze the multi-scale and multi-physics interaction between the cardiovascular flow hemodynamics, the cardiac function, and the peripheral circulation. The Meshless solver is based on localized RBF collocations at scattered data points which allows for automation of the model generation via CAD integration. The time-accurate incompressible flow hemodynamics are addressed via a pressure-velocity correction scheme where the ensuing Poisson equations are accurately and efficiently solved at each time step by a Dual-Reciprocity Boundary Element method (DRBEM) formulation that takes advantage of the integrated surface discretization and automated point distribution used for the Meshless collocation. The local hemodynamics are integrated with the peripheral circulation via compartments that account for branch viscous resistance $(\mathrm{R})$, flow inertia $(\mathrm{L})$, and vessel compliance (C), namely RLC electric circuit analogies. The cardiac function is modeled via time-varying capacitors simulating the ventricles and constant capacitors simulating the atria, connected by diodes and resistors simulating the atrioventricular and ventricular-arterial valves. This multi-scale integration in an in-house developed computational tool opens the possibility for model automation of patient-specific anatomies from medical imaging, elastodynamics analysis of vessel wall deformation for fluid-structure interaction, automated model refinement, and inverse analysis for parameter estimation.
\end{abstract}

Keywords: Lumped-Parameter Model, Multi-scale CFD, Meshless Methods, DRBEM

\section{INTRODUCTION}

Multidimensional hemodynamics analysis of the cardiovascular system usually couples two types of computational models: a 2- or 3-dimensional section of interest where a full-detail hemodynamic analysis can be performed, and a 0-dimensional lumped-parameter model (LPM) of the peripheral circulation [1-4], as shown in Fig. 1. Current state-of-the-art couples, either loosely or strongly, the solution from in-house or commercial fluid solvers, for example, StarCCM+ or Fluent, with a LPM using in scripts to carry information about the boundary conditions back and forth. This is done at every iteration until the resultant parameters reach convergence [5-9]. Solutions such are typically sought for the pressure and flow dynamics, pulmonary artery pressure and flow, coronary artery flow and pressure, and the ratio of pulmonary blow flow and systemic blood flow [4].

The analysis of the full hemodynamics of the aortic arch is proposed using a meshless methods solver. A localized Radial-Basis Function Collocation Meshless Method (LRC-MM) addresses the high computational expense issue that would occur if a global RBF interpolation were to be used [10-12]. When decoupling of the incompressible governing equations of fluid flow, the resultant Poisson-like equations that arise from the solution of the pressure field at every time step are solved by a Dual Reciprocity Boundary Element Method (DR-BEM) which ensures high stability and accuracy. DR-BEM transfers the generation term of the resultant Poisson equation to the boundary via an RBF expansion [13, 14]. Since there is an existing point distribution from the LRC-MM, it can be used again when solving the boundary-only integration and determining the internal solution [15]. 


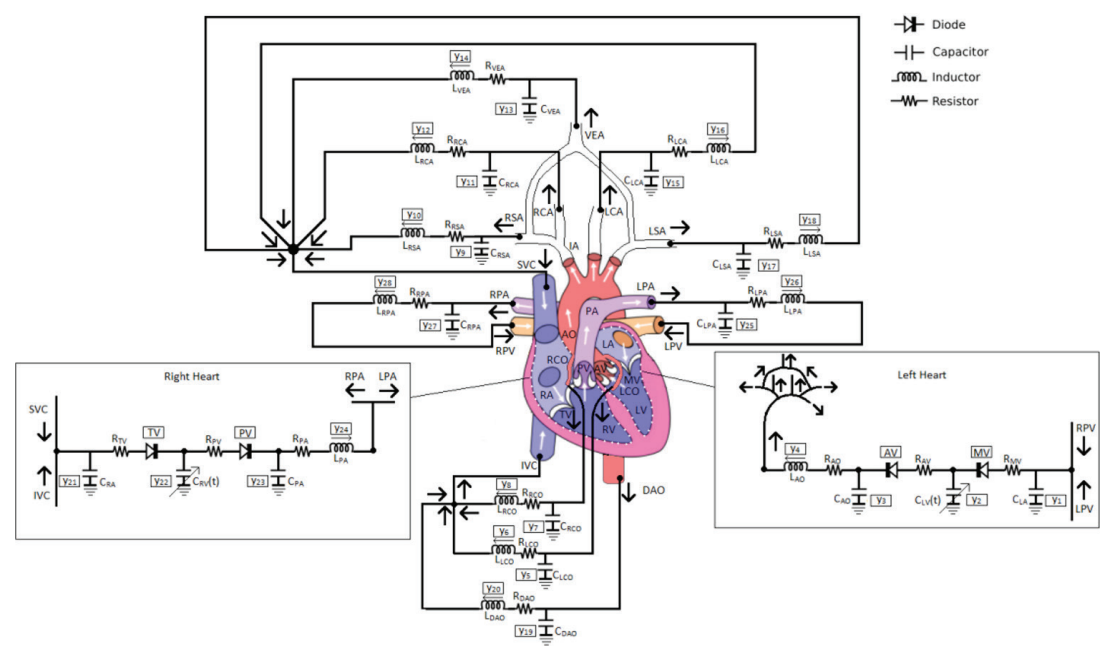

Figure 1: Visual representation of the integrated LRC-MM and LPM.

Beyond the region of interest where the details of the hemodynamics are to be resolved, the aortic bed for instance, the peripheral circulation can be simulated using a 0D lumped parameter model based on an electric analogy to fluid flow. Utilizing the Windkessel compartment model, the LPM is used to reproduce the impedance that the peripheral bed presents to the inlet and outlets of mutli-dimensional region of interest via a combination of effective viscous resistance to flow, compliance, and convective acceleration or inertance experienced by the flow in the peripheral vasculature [16]. In this instance, the multi-compartment model used is an RLC (resistance, inductance and capacitance) arrangement that can be used to flexibly model the entire network. Instead of treating the entire network as a single block, the different segments allow for the flow and pressure in different sections to be calculated [17]. The heart is also represented in this system though a combination of diodes that model heart valves, resitances and inductances, as well as a time-varying elastance function (inverse of the compliance) that drives the circuit. The latter serves as the analogue of the elastance of the myocardium [18].

\section{3-D MESHLESS SOLVER APPROACH}

The hemodynamics of the aorta will be solved using a 3-D meshless solver for the Navier-Stokes equations [10].

$$
\begin{aligned}
& \nabla \cdot \vec{V}=0 \\
& \rho \frac{\partial \vec{V}}{\partial t}+\rho(\vec{V} \cdot \nabla) \vec{V}=\mu \nabla^{2} \vec{V}-\nabla p+\vec{f}
\end{aligned}
$$

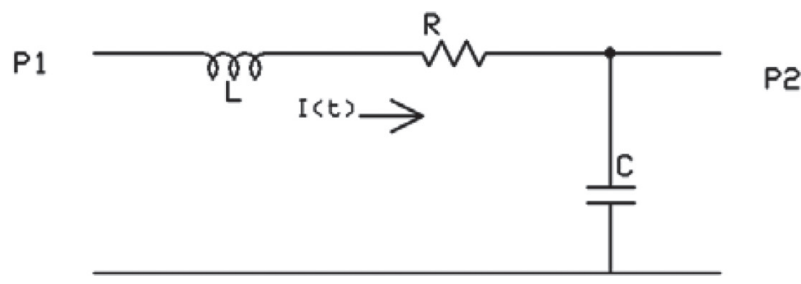

Figure 2: Single RLC compartment used in LPM. 
For this incompressible case, the density, $\rho$, is constant [10]. The field variables of interest are the velocity, $\vec{V}(x, t)$ and the pressure $p(x, t)$. Therefore, the velocity advances as:

$$
\begin{array}{r}
\vec{V}^{n+1}(x)=\vec{V}^{n}(x)+\Delta t\left[\frac{\mu}{\rho} \nabla^{2} \vec{V}^{n}(x)-\left(\vec{V}^{n}(x) \cdot \nabla\right) \vec{V}^{n}(x)\right. \\
\left.-\frac{1}{\rho} \nabla p^{n+1}(x)+\frac{1}{\rho} \vec{f}^{n}(x)\right]
\end{array}
$$

Given that there is no explicit pressure equation, a pressure correction field is introduced:

$$
p^{n+1}(x)=p^{n}(x)+\phi^{n}(x)
$$

Substituting (3) into (2), eventually leads to a Poisson equation whose solution results in the pressure correction, $\phi$, from (3) [10]:

$$
\nabla^{2} \phi^{n}(x)=\frac{\rho}{\Delta t}\left(\nabla \cdot \vec{V}^{*}(x)\right)
$$

\subsection{Dual Reciprocity Boundary Element Method (DR-BEM)}

Given the need to solve (4) for the field variable, the pressure correction $\phi(x)$, a boundary element method (BEM) approach is proposed through the formulation of a boundary integral equation (BIE). The purpose of the BIE, obtained through an RBF expansion, is to obtain a BEM solution to the necessary domain integration that results from Green's free-space solution [10]. For the Poisson equation in (4), the right hand side is known and non-homogeneous, $B(x)$. Considering a weight function, $G(x, \zeta)$, applying Green's 2 nd identity to the resultant equation while letting $G(x, \zeta)$ be Green's free-space solution of the Laplace equation that solved the adjoint equation, $\nabla^{2} G(x, \zeta)=-\delta(x, \zeta)$, where $\delta(x, \zeta)$ is the Dirac Delta function. For example, in 2D the free-space solution is $G(x, \zeta)=-\ln (r) / 2 \pi s$, and there results an integral equation in terms of the field variable:

$$
c(\zeta) \phi^{n}(\zeta)+\oint_{\Gamma} \phi^{n}(\zeta) H(x, \zeta) d \Gamma=\oint_{\Gamma} q^{n}(x) G(x, \zeta) d \Gamma+b(\zeta)
$$

This solution still contains terms that require a domain integral:

$$
\begin{aligned}
q^{n}(x)=\frac{\partial \phi^{n}(x)}{\partial n} & b(\zeta)=\int_{\Omega} B(x) G(x, \zeta) d \Omega \\
H(x, \zeta)=\frac{\partial G(x, \zeta)}{\partial n} & c(\zeta)=\int_{\Omega} \delta(x, \zeta) d \Omega
\end{aligned}
$$

However, the parameter $c(\zeta)$ is 1 inside the domain and 0.5 at the boundary. $b(\zeta)$ still contains a domain integral but with an RBF expansion of $B(x)$, using polyharmonic RBFs, eventually leads to its boundary form of:

$$
\begin{aligned}
b_{i} & =\sum_{k=1}^{N} \alpha_{k}\left[\oint_{\Gamma} p_{k}(x) G\left(x, \zeta_{i}\right) d \Gamma-\oint_{\Gamma} u_{k}(x) H\left(x, \zeta_{i}\right) d \Gamma-c\left(\zeta_{i}\right) u_{k}\left(\zeta_{i}\right)\right] \\
p_{k}(x) & =\frac{\partial u_{k}(x)}{\partial n} \\
H(x, \zeta) & =\frac{\partial G(x, \zeta)}{\partial n}
\end{aligned}
$$


And the BIE can be fully written in terms of boundary integrals:

$$
c_{i} \phi_{i}^{n}+\sum_{j=1}^{N_{B}} \hat{H}_{i j} \phi_{j}^{n}=\sum_{j=1}^{N_{B}} G_{i j} q_{j}^{n}+\sum_{k=1}^{N} \alpha_{k}\left[\sum_{j=1}^{N_{B}} G_{i j} p_{j}^{k}-\sum_{j=1}^{N_{B}} H_{i j} u_{j}^{k}\right]
$$

From the boundary solution, the field variable can be calculated at point inside the domain without the use of domain integrals. In addition, any derivative of the field variable can now be determined through the discretized form of the BIE.

\section{LUMPED-PARAMETER MODEL}

In the RLC compartment used to simulate the state of a set of vessels each component represents a physical characteristic: a resistance (R), accounts for viscous drag, an inductance (L), for flow inertia, and a capacitance $(\mathrm{C})$, for vascular wall compliance. In the case of relevant variables, pressure $(\mathrm{P})$ is voltage $(\mathrm{V})$ and flow rate $(\mathrm{Q})$ is current $(\mathrm{I})$ [19], [20]. A time-varying capacitance, $C(t)$, represents the contractile behavior of the ventricle. A diode acts as the corresponding cardiac valve and insures unidirectional flow over the relaxation and contraction phases of the cardiac cycle [18, 21].

Given the incompressible Navier-Stokes equation from (1) and assuming fully developed axial flow and neglecting the external body force, the pressure gradient results in:

$$
\frac{\partial p(z, t)}{\partial z}=\frac{\mu}{r} \frac{\partial}{\partial r}\left[r \frac{\partial u(r, t)}{\partial r}\right]-\rho \frac{\partial u(r, t)}{\partial t}
$$

The axial velocity profile in the circular cross-section of radius $\mathfrak{R}$, assuming laminar flow, is:

$$
u(r, t)=2 \bar{u}(t)\left[1-\left(\frac{r}{\mathfrak{R}}\right)^{2}\right]
$$

Where $\bar{u}(t)$ is the time-varying cross-section-averaged mean axial velocity. After evaluating and integrating over the cross-section:

$$
\frac{\partial p(z, t)}{\partial z} A_{c}=-\frac{8 \mu}{\mathfrak{R}^{2}} Q(t)-\rho \frac{d Q(t)}{d t}
$$

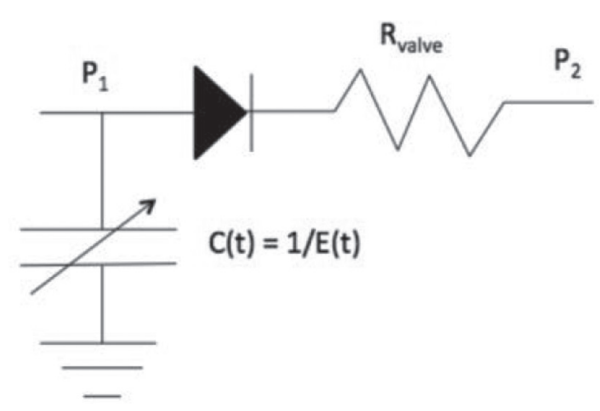

Figure 3: Electrical compartment containing the time-varying compliance. 
Where $A_{c}$ is the cross-section and $Q(t)$ is the volumetric flow rate. The resultant pressure drop, $\Delta p$, along a distance, $\Delta p$, is:

$$
\Delta p(t)=\left(\frac{8 \mu l}{\mathfrak{R}^{2} A_{c}}\right) Q(t)+\left(\frac{\rho l}{A_{c}}\right) \frac{d Q(t)}{d t}
$$

The analogy of the cardiovascular behavior and the electrical components is given in Table 1 [20]. The heaviside step function is used to represent the behavior of the heart valves, where the pressure drop controls the valve opening or closing depending on a pressure difference $\Delta P$ thus allowing the blood to flow in only one direction.

Since the total voltage drop through a resistor-inductor is:

$$
\Delta v(t)=R i(t)+L \frac{d i(t)}{d t}
$$

Yielding the actual resistance and inductance in hydraulic flow terms from (12):

$$
R=\frac{8 \mu l}{\mathfrak{R}^{2} A_{c}} \quad L=\frac{\rho l}{A_{c}}
$$

\subsection{The Heart in LPM}

Elastance is related to the ventricle pressure and volume according to [18]:

$$
E(t)=\frac{P(t)}{V(t)-V_{0}}
$$

Where $V_{0}$ is the volume of the ventricle at zero pressure. Mathematically, the time varying elastance is given by:

$$
E(t)=\left(E_{\max }-E_{\min }\right) E_{n}\left(t_{n}\right)+E_{\min }
$$

The ventricular function can be simulated using the "double hill function" that represents the normalized elastance $E_{n}\left(t_{n}\right)[21]$ :

$$
E_{n}\left(t_{n}\right)=1.55\left[\frac{\left(\frac{t_{n}}{0.7}\right)^{1.9}}{1+\left(\frac{t_{n}}{0.7}\right)^{1.9}}\right]\left[\frac{1}{1+\left(\frac{t_{n}}{1.17}\right)^{21.9}}\right]
$$

Table 1. Cardiovascular behavior and corresponding electrical analogy.

\begin{tabular}{lll}
\hline Cardiovascular Behavior & Electrical Component & Relationship \\
\hline Vessel Resistance & Resistor & $\Delta P=Q R$ \\
Blood Vessel Compliance & Capacitor & $Q=C d P / d t$ \\
Flow Inertia & Inductor & $\Delta P=L d Q / d t$ \\
Heart valve & Diode & $Q=\left(\Delta P / R_{\text {valve }}\right) H(\Delta P)$ \\
\hline
\end{tabular}


Where: $t_{n}=t / T_{\max }$ and $T_{\max }=0.2+0.15 t_{c}$ such that $t_{c}$ is the cardiac cycle interval in seconds.

For a healthy adult left ventricle: $E_{\max }=2 \mathrm{mmHg} / \mathrm{ml}$ and $E_{\min }=0.06 \mathrm{mmHg} / \mathrm{ml}$, [21]. The time-dependant elastance is the reciprocal of the time-varying capacitance, $E(t)=1 / C(t)$ and are shown in Fig. 4 for a heart rate, HR, of 60 beats per minute (bpm).

\section{COUPLING OF LRC-MM AND LPM}

The multi-scale analysis requires the full system to be integrated such that the LPM is connected to the LRC-MM in order to have a closed-loop system. At points of coupling, relevant flow information will have to be converted from 3D- to 0D-analysis and back to 3D in order to complete the close-loop as in Fig. 1. The concept behind this coupling process is illustrated in Fig. 5.

The proposed approach is different on whether coupling is from the meshless solver to the LPM or vice versa. The two relevant parameters used are the local pressure and the volumetric flow rate.

- 3D-0D connection: the average values from the nodes at the cross-sectional surface used as input for the $0 \mathrm{D}$ compartment(s).

- 0D-3D connection: due to the assumption of Poiseuille flow, as mentioned earlier, the velocity from the LPM, $u_{\text {mean }}$, can be used to calculate the parabolic shape of flow field across the nodes on the surface. The local pressure from the LPM is transferred uniformly across the surface.

\section{PRELIMINARY RESULTS}

RBF-based Meshless solvers have been able to provide reliable simulations for several applications $[11,12,15]$. As a preliminary test to the multi-scale algorithms, a simplified model of
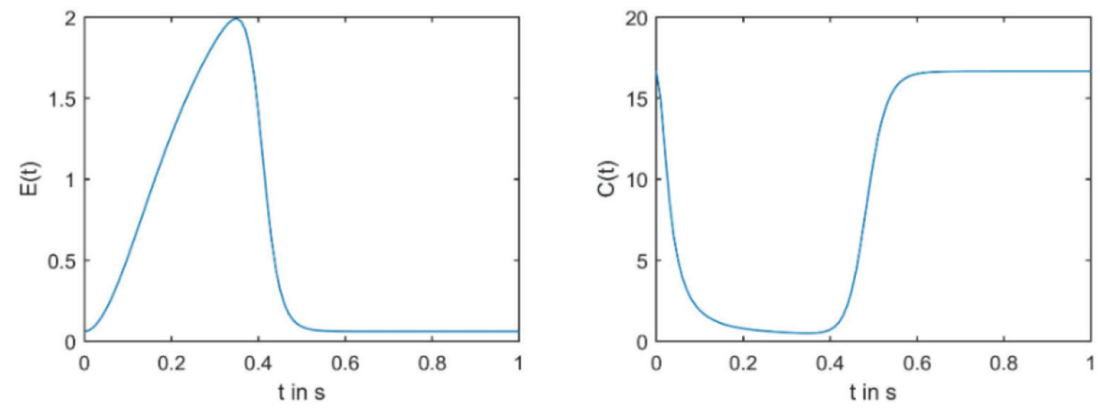

Figure 4. Elastance, $E(t)$ and ventricular compliance, $C(t)$, for a healthy heart at $60 \mathrm{bpm}$.

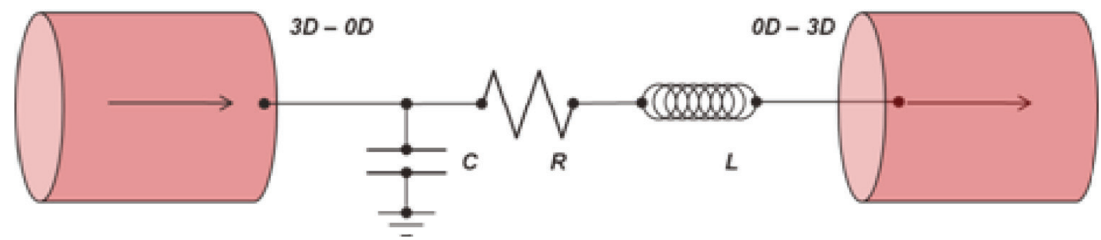

Figure 5: Coupling between an RLC compartment and 3D LRC-MM analysis. 
a bulging and bifurcating vessel is configured to be loosely coupled with the LPM. The volumetric flow rate resulting from the LPM is passed at very time step as the inlet condition to the meshless model. The fluid properties of blood are used with the density $\rho=1,060 \mathrm{Kg} / \mathrm{m}^{3}$ and the viscosity $\mu=3.5 \cdot 10^{-3} \mathrm{Kg} / \mathrm{m} \cdot \mathrm{s}$.

The LPM is tested using a simplified model of the cardiovascular system, accounting for the compliance of the left atrium $\left(C_{A}\right)$, mitral valve $\left(D_{M}, R_{M}\right)$, left ventricle, driving the system with the time-varying capacitance $(C(t))$, aortic valve $\left(D_{A}, R_{A}\right)$, aorta compliance and characteristic resistance and inductance $\left(C_{A O}, R_{A O}, L_{A O}\right)$, and systemic circulation compliance and resistance $\left(C_{S}, R_{S}\right)[18,21]$. The values associated with each of the resistances, compliances, or inductances, are well documented in the literature [21-23]. The arrangement using RLC compartments and a variable capacitance for the left ventricular compliance is shown in Fig. 6.

The LPM is solved for a healthy adult at $60 \mathrm{bpm}$ over a period of 5 seconds. The resultant pressures at different locations: the left atrium (LA), $P_{L A}$, the left ventricle (LV), $P_{L V}$, the aorta (AO), $P_{A O}$, and the systemic pressure, $P_{S Y S}$ are shown in Fig. 7.

The meshless model consists of a bulging and bifurcating vessel with an inlet hydraulic diameter of $3 \mathrm{~cm}$ bulging to $5 \mathrm{~cm}$ after $10 \mathrm{~cm}$ of entry length. Bifurcation occurs after $30 \mathrm{~cm}$ and the outlets have hydraulic diameters of $1.5 \mathrm{~cm}$ and $2 \mathrm{~cm}$. The overall length of the model

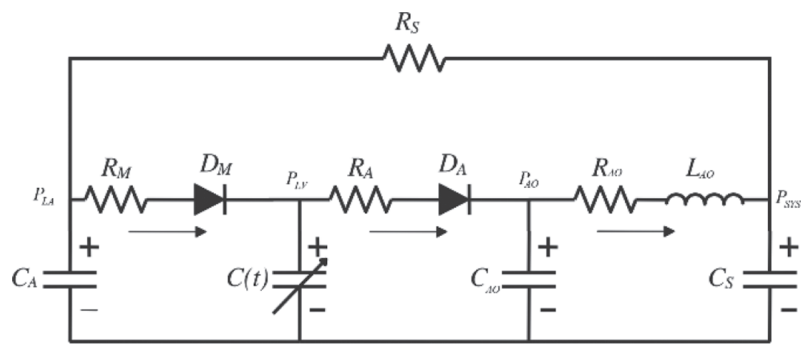

Figure 6: Simplified Cardiovascular circuit model [18].

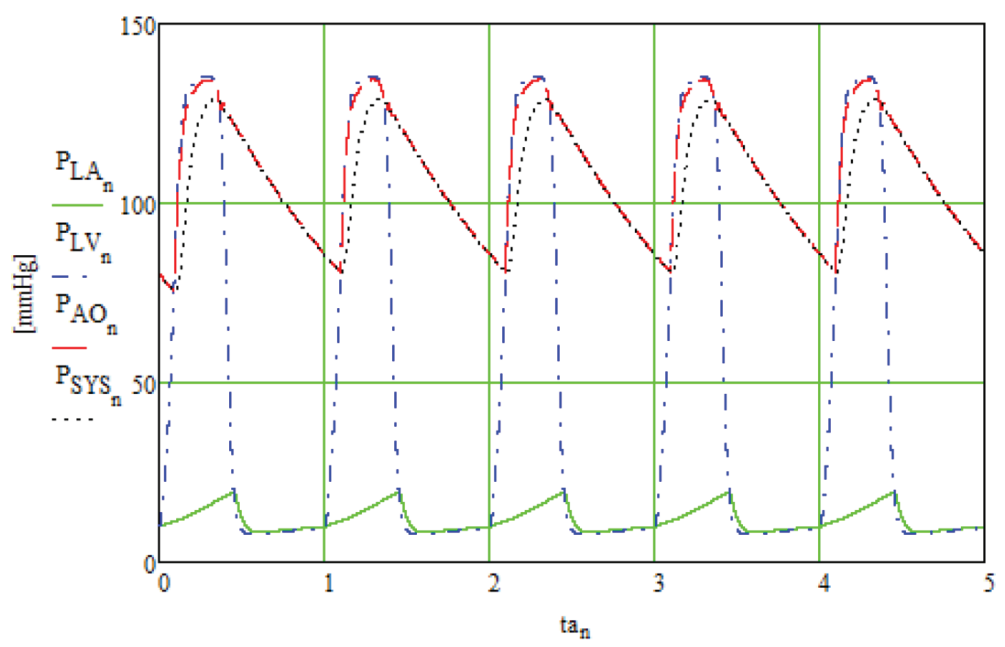

[s]

Figure 7: Pressure and Volumetric Flow after 5 cycles in LPM. 


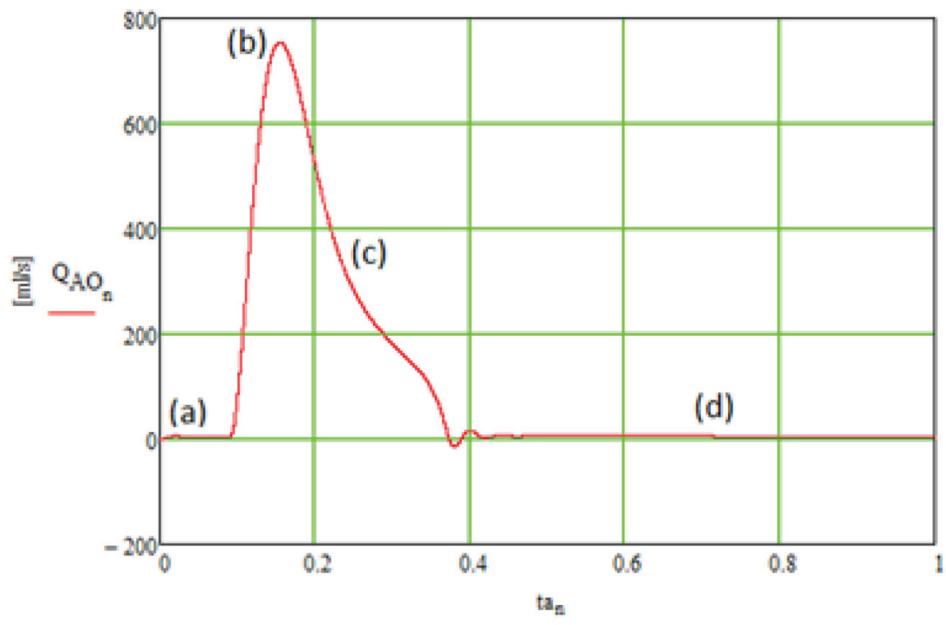

[s]

Figure 8: Volumetric flow rate for a single cycle of LPM.

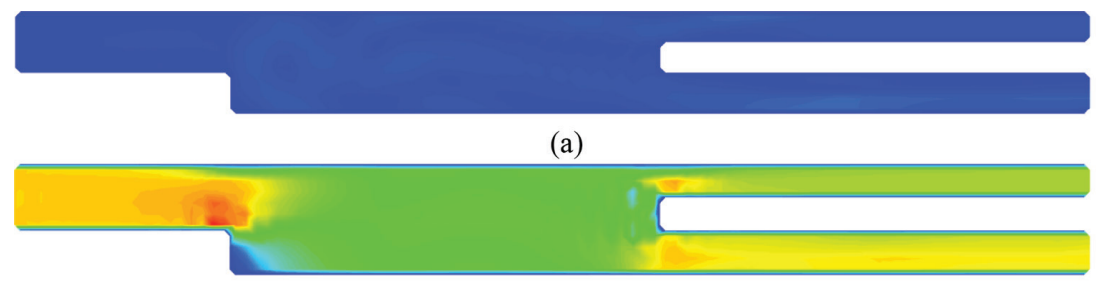

(b)

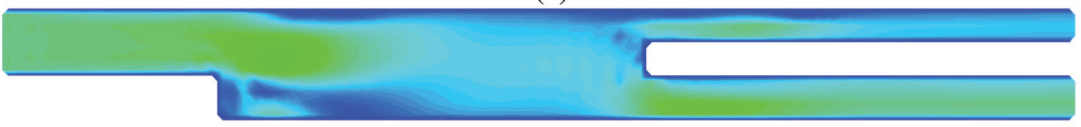

(c)

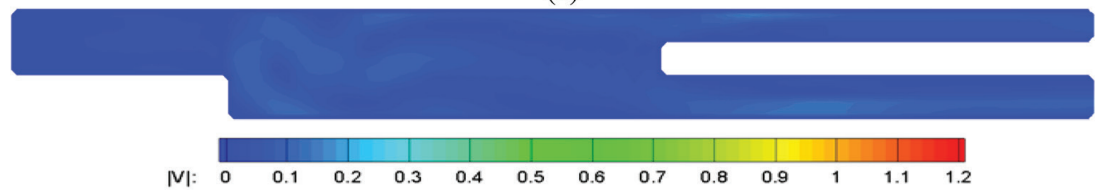

(d)

Figure 9: Velocity contour plots at representative locations throughout heart cycle: (a) onset of systole, (b) peak systole, (c) end of systole, and (d) diastole.

is $50 \mathrm{~cm}$. The resulting LPM flow rates yield an average representative Reynolds number around 1,200. Representative locations throughout the cardiac cycle, as shown in Fig. 8 for the LPM-generated flow rates, are selected to plot the corresponding velocity contours as shown in Fig. 9, for the last of the 5 generated cardiac cycles. 


\section{CONCLUSION}

The proposed approach to address the multi-scale analysis of cardiovascular flow utilizes a Localized Radial-Basis Function Collocation Meshless Method (LRC-MM) solver aided by a stable and robust Dual-Reciprocity Boundary Element Method (DR-BEM) solver to address the pressure field at every time step of the solution for the local hemodynamics at a $3 \mathrm{D}$ scale. The coupled LRC-MM/DR-BEM approach is implemented over the same boundary discretization and internal point distribution which is automatically generated and allows for direct CAD integration. The peripheral circulation and heart function is represented in a $0 D$ scale by electrical circuit analogies of the Lumped Parameter Models (LPM) where the viscous drag is represented by a resistor (R), the flow inertia is represented by an inductor (L), the wall compliance is represented by a capacitor (C), and the heart valve is represented by a diode (D). This electrical elements are lumped together in compartments representing the different branches of the cardiovascular circuit. The LRC-MM/DR-BEM and OD LPM are effectively coupled to form a multi-scale closed circuit of the cardiovascular system. The in-house computational tool will further enable analysis of patient-specific geometry, fluid-structure interaction analysis of vessel wall deformation, automated model refinement. LPM model parameters can be obtained on a patient specific basis given measured pressure and flow waveforms on the basis for inverse analysis.

\section{REFERENCES}

[1] Migliavacca, F., Pennati, G., Dubini, G., Fumero, R., Pietrabissa, R., Urcelay, G., Bove, E.L., Hsia, T-Y. \& de Leval, M.R., Modeling of the Norwood circulation: effects of shunt size, vascular resistances, and heart rate. American Journal of Physiology - Heart and Circulatory Physiology, 280(5), pp. H2076-H2086, 2001.

[2] Migliavacca, F., Balossino, R., Pennati, G., Dubini, G., Hsia, T-Y., de Leval, M.R. \& Bove, E.L., Multiscale modelling in biofluidynamics: application to reconstructive paediatric cardiac surgery. Journal of Biomechanics, 39(6), pp. 1010-1020, 2006. https://doi.org/10.1016/j.jbiomech.2005.02.021

[3] DeCampli, W.M., Argueta-Morales, I.R., Divo, E. \& Kassab, A.J., Computational fluid dynamics in congenital heart disease. Cardiology in the Young, 22(06), pp. 800-808, 2012. https://doi.org/10.1017/s1047951112002028

[4] Hsia, T-Y., Cosentino, D., Corsini, C., Pennati, G., Dubini, G., Migliavacca, F. \& Modeling of Congenital Hearts Alliance (MOCHA) Investigators. Use of mathematical modeling to compare and predict hemodynamic effects between hybrid and surgical Norwood palliations for hypoplastic left heart syndrome. Circulation, 124(11 Suppl), pp. S204-S210, 2011. https://doi.org/10.1161/circulationaha.110.010769

[5] Ceballos, A., A Coupled CFD-Lumped Parameter Model of the Human Circulation: Elucidating the Hemodynamics of the Hybrid Norwood Palliative Treatment and Effects of the Reverse Blalock-Taussic Shunt Placement and Diameter. UCF, Orlando, Florida, 2015.

[6] Ceballos, A., Argueta-Morales, I.R., Divo, E., Osorio, R., Caldarone, C.A., Kassab, A.J. \& DeCampli, W.M., Computational analysis of hybrid norwood circulation with distal aortic arch obstruction and reverse Blalock-Taussig Shunt. The Annals of Thoracic Surgery, 94(5), pp. 1540-1550, 2012.

https://doi.org/10.1016/j.athoracsur.2012.06.043 
[7] Prather, R., A Multi-Scale CFD Analysis of Patient-Specific Geometries to Tailor LVAD Cannula Implantation Under Pulsatile Flow Conditions: an investigation aimed at reducing stroke incidence in LVADs. University of Central Florida, Orlando, 2015.

[8] Prather, R.O., Kassab, A., Ni, M.W., Divo, E., Argueta, I.R. \& DeCampli, W.M., Multi-scale pulsatile CFD modeling of thrombus transport in a patient-specific LVAD implantation. International Journal of Numerical Methods for Heat \& Fluid Flow, 27(5), pp. 1022-1039, 2017.

https://doi.org/10.1108/hff-10-2016-0378

[9] Quarteroni, A., Ragni, S. \& Veneziani, A., Coupling between lumped and distributed models for blood flow problems. Computing and Visualization in Science, 4(2), pp. 111-124, 2001.

https://doi.org/10.1007/s007910100063

[10] Pepper, D.W., Kassab, A. \& Divo, E., An Introduction to Finite Element, Boundary Element, and Meshless Methods with Applications to Heat Transfer and Fluid Flow, ASME Press, New York, 2014.

[11] Divo, E. \& Kassab, A.J., Localized meshless modeling of natural-convective viscous flows. Numerical Heat Transfer, Part B: Fundamentals, 53(6), pp. 487-509, 2008. https://doi.org/10.1080/10407790802083190

[12] Divo, E. \& Kassab, A.J., An efficient localized radial basis function meshless method for fluid flow and conjugate heat transfer. Journal of Heat Transfer, 129(2), pp. 124-136, 2006.

https://doi.org/10.1115/1.2402181

[13] Wrobel, L.C. \& Brebbia, C.A., The dual reciprocity boundary element formulation for nonlinear diffusion problems. Computer Methods in Applied Mechanics and Engineering, 65(2), pp. 147-164, 1987.

https://doi.org/10.1016/0045-7825(87)90010-7

[14] Divo, E. \& Kassab, A.J., Transient non-linear heat conduction solution by a dual reciprocity boundary element method with an effective posteriori error estimator. Heat Transfer, 1, pp. 77-86, 2004.

https://doi.org/IMECE2004-59262

[15] Bueno, L.A., Divo, E.A. \& Kassab, A.J., A coupled localized Rbf Meshless/DRBEM formulation for accurate modeling of incompressible fluid flows. International Journal of Computational Methods and Experimental Measurements, 5(3), pp. 359-368, 2017. https://doi.org/10.2495/cmem-v5-n3-359-368

[16] Kind, T., Faes, T.J.C., Lankhaar, J.W., Vonk-Noordegraaf, A. \& Verhaegen, M., Estimation of three- and four-element windkessel parameters using subspace model identification. IEEE Transactions on Biomedical Engineering, 57(7), pp. 1531-1538, 2010. https://doi.org/10.1109/tbme.2010.2041351

[17] Kokalari, I., Karaja, T. \& Guerrisi, M., Review on lumped parameter method for modeling the blood flow in systemic arteries. Journal of Biomedical Science and Engineering, 06(01), pp. 92-99, 2013.

https://doi.org/10.4236/jbise.2013.61012

[18] Faragallah, G., Wang, Y., Divo, E. \& Simaan, M., A new control system for left ventricular assist devices based on patient-specific physiological demand. Inverse Problems in Science and Engineering, 20(5), pp. 721-734, 2012. https://doi.org/10.1080/17415977.2012.667092 
[19] Formaggia, L. \& Veneziani, A., Reduced and multiscale models for the human cardiovascular system. Lecture Notes VKI Lecture Series, 7, 2003.

[20] Creigen, V., Ferracina, L., Hlod, A., van Mourik, S., Sjauw, K., Rottschäfer, V., Vellekoop, M. \& Zegeling, P., Modeling a heart pump. European Study Group Mathematics with Industry, Utrecht, p. 7, 2007.

[21] Simaan, M.A., Ferreira, A., Chen, S., Antaki, J.F. \& Galati, D.G., A dynamical state space representation and performance analysis of a feedback-controlled rotary left ventricular assist device. IEEE Transactions on Control Systems Technology, 17(1), pp. 15-28, 2009.

https://doi.org/10.1109/tcst.2007.912123

[22] Yu, Y-C., Boston, J.R., Simaan, M.A. \& Antaki, J.F., Minimally invasive estimation of systemic vascular parameters. Annals of Biomedical Engineering, 29(7), pp. 595-606, 2001.

https://doi.org/10.1114/1.1380420

[23] Yu, Y-C., Boston, J.R., Simaan, M.A. \& Antaki, J.F., Estimation of systemic vascular bed parameters for artificial heart control. IEEE Transactions on Automatic Control, 43(6), pp. 765-778, 1998.

https://doi.org/10.1109/9.679017 